\title{
COVID-19 vaccination for children: may be necessary for the full eradication of the disease
}

(c) The Author(s), under exclusive licence to the International Pediatric Research Foundation, Inc 2021

Pediatric Research (2021) 90:1102-1103; https://doi.org/10.1038/s41390021-01643-y

After years of anti-vaccination campaigns, ${ }^{1}$ a small measles pandemic occurred in Europe in 2017, due to suboptimal vaccination coverage in the population (i.e., $<80 \%$ ). In that year, $>15,000$ cases of measles were reported, in particular in Romania, Italy, and Greece, especially among children. Due to this lack of trust and to ineffective vaccination schedules, at least 50 (preventable) deaths occurred in this age range. ${ }^{2}$

These numbers may appear very limited in comparison to the dramatic morbidity and death toll related to COVID-19 pandemic, but in some way this dangerous precedent may permit us to forecast what could happen in the next months in a world with children not vaccinated against COVID-19.

In the last months, the most significant contribution against COVID-19 spreading came from the extraordinary (and effective) vaccination campaign, which, together with lockdown politics, physical distancing, and other protective measures, have dramatically lowered the infectious and mortality rates. Nevertheless, this massive vaccination campaign still remains "on-label" for children above the age of 12 years, and, at the present day, only limited vaccination campaigns have been performed in (healthy) children below the age of 20 years.

This lack of attention for the youngest-aged people is motived by the fact that COVID-19 infection in childhood has a wellrecognized benign and limited course, with low to moderate fever, influenza-like, and/or gastrointestinal manifestations. ${ }^{3}$ However, in a very limited portion of young patients, COVID-19 may be associated with a subsequent serious condition, firstly reported as "Kawasaki-like disease," and later recognized as "multisystem inflammatory syndrome in children" (MIS-C). ${ }^{4}$ This syndrome involves pediatric patients of all ages (median age of 8-9 years, half of the cases being comprised between 5 and 13 years) and, notably, in most of these cases it is not preceded or accompanied by preexisting medical conditions. It is usually developed 2-4 weeks after a severe acute respiratory syndrome coronavirus 2 (SARS-CoV-2) infection or a close contact with an infected person, and is characterized by febrile illness of short duration with prominent gastrointestinal, cardiac, and hematologic manifestations, including vasoplegic shock, acute myocarditis, and coronary artery aneurysms. At least half of these patients may require intensive care treatment, with mortality ranging from 0 to $4 \%{ }^{4,5}$ It is still unclear why only some pediatric patients develop this severe condition, but a racial predisposition has been reported in the United States (63\% of patients were Hispanic or Black) and the male sex is more often involved $(60 \%) .^{5}$ Its incidence is very limited, with $\sim 3500$ cases reported in the USA till May 2021. ${ }^{5}$

As it has been difficult to justify a COVID-19 vaccine in children for a disease that appears to be mild in most cases, particularly because children play a minimal role in spreading the infection to others, ${ }^{6}$ only a minority of trials have been performed to test the efficacy and safety of COVID-19 vaccination in children and a massive vaccination campaign for this age range is still far to come. Importantly, each vaccine must be tested and proven to be safe before being administered to children, and protocols in this age range may be ethically difficult to be performed, ${ }^{6}$ considering also that vaccination for children is not primarily performed for their self-protection but for that of the community, mainly the elderly or high-risk individuals.

Even limiting the vaccination only to adult people, it is likely that a "herd immunity" will be reached by the end of the next summer in most of the Western Countries, and for this reason, they are progressively approaching lightening of the physical distancing measures and lockdown politics. It should be also considered that the exceptional results on the usefulness and safety of the messenger RNA vaccines in children aged 12-15 years will increase the amount of vaccinated people. ${ }^{7,8}$ However, it is presumable that SARS-CoV-2 will continue to spread, even with a lower incidence, and is limited only to the non-vaccinated people (in particular children younger than 12 years), similarly to what happened with measles in 2017, when a decrease of vaccinated children under the critical threshold of $75-80 \%$ has brought to a significant increase of admissions and fatalities. ${ }^{2}$ It is likely that in the next months, when virtually no children below the age of 12 years will be vaccinated against SARS-CoV-2, some measures will be still needed for young children, like maintaining physical distancing and wearing individual protection at school, as well during sport and leisure activities in a closed environment, with problematic psychological and physical consequences. ${ }^{9}$

Governments and drug authorities should encourage studies for testing the safety and efficacy of COVID-19 vaccination in children, considering also that in many underdeveloped countries the proportion of population $<15$ years of age is dramatically higher than in richest nations and that only a worldwide vaccination campaign can efficaciously contain the disease, also by limiting the possibility of the development of new SARS-CoV-2 variants. Lastly, if the vaccine will be proven to be safe and efficacious in children, educational campaigns on the utility and safety of vaccination amongst children should be promoted, this in order to avoid suboptimal coverage and to prevent COVID-19- as well as MIS-C-related death among children.

Andrea D. Praticò ${ }^{1 凶}$ and Martino Ruggieri ${ }^{1}$
${ }^{1}$ Unit of Rare Diseases of the Nervous System in Childhood,
Department of Clinical and Experimental Medicine, University of
Catania, Catania, Italy. ${ }^{\bowtie}$ email: andrea.pratico@unict.it

\section{REFERENCES}

1. Johnson, N. F. et al. The online competition between pro- and anti-vaccination views. Nature 582, 230-33 (2020). 
2. European Centre for Disease Prevention and Control - News \& events. Measles cases in the EU treble in 2017, outbreaks still ongoing. https://www.ecdc.europa. eu/en/news-events/measles-cases-eu-treble-2017-outbreaks-still-ongoing (2021).

3. Mehta, N. S. et al. SARS-CoV-2 (COVID-19): What do we know about children? A systematic review. Clin. Infect. Dis. 71, 2469-79 (2020).

4. Radia, T. et al. Multi-system inflammatory syndrome in children \& adolescents (MIS-C): a systematic review of clinical features and presentation. Paediatr. Respir. Rev. S1526-0542, 30117-2 (2020).

5. Centers for Diseases Controls and Prevention. MIS and COVID-19. https://www.cdc. gov/mis-c/cases/index.html (2021).

6. Opel, D. J., Diekema, D. S. \& Ross, L. F. Should we mandate a COVID-19 vaccine for children? JAMA Pediatr. 175, 125-6 (2021).

7. Wallace, M. et al. The Advisory Committee on Immunization Practices' Interim Recommendation for Use of Pfizer-BioNTech COVID-19 Vaccine in Adolescents Aged 12-15 Years - United States, May 2021. MMWR 70, 749-52 (2021).
8. Tanne, J. H. Covid-19: FDA authorises Pfizer vaccine for children 12-15. BMJ 11, 1204 (2021).

9. Praticò, A. D. COVID-19 pandemic for pediatric health care: disadvantages and opportunities. Pediatr. Res. 89, 709-10 (2021).

\section{ADDITIONAL INFORMATION}

Correspondence and requests for materials should be addressed to A.D.P.

Reprints and permission information is available at http://www.nature.com/ reprints

Publisher's note Springer Nature remains neutral with regard to jurisdictional claims in published maps and institutional affiliations. 